\title{
Nanolipoprotein particles (NLPs) as a delivery platform for Fab based therapeutics
}

Martine Darwish+, Whitney Shatz ${ }^{+}$, Brandon Leonard, Kelly Loyet, Kathy Barrett, Janice L. Wong, Hong Li, Ryan Abraham, May Lin, Yvonne Franke, Christine Tam, Kyle Mortara, Inna Zilberleyb and Craig Blanchette*

+ Authors contributed equally

* Corresponding author

Genentech, South San Francisco, CA 94088

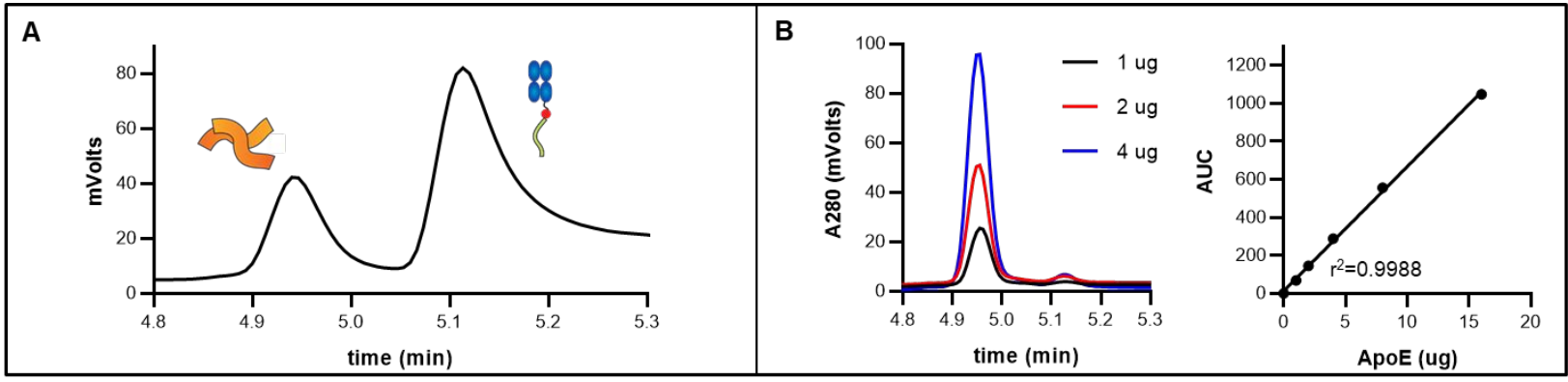

Figure S1. Quantifying the protein composition of Fab-NLP conjugates. A. HPLC trace of a Fab-NLP conjugate. Two peaks were observed at $4.95 \mathrm{~min}$ and $5.15 \mathrm{~min}$ that correspond to apoE422k and Fab, respectively. B. HPLC traces of apoE422k at different injection amounts and the corresponding standard curve. The apoE422k standard curve was used to determine the apoE422k concentration of the Fab-NLP conjugate.

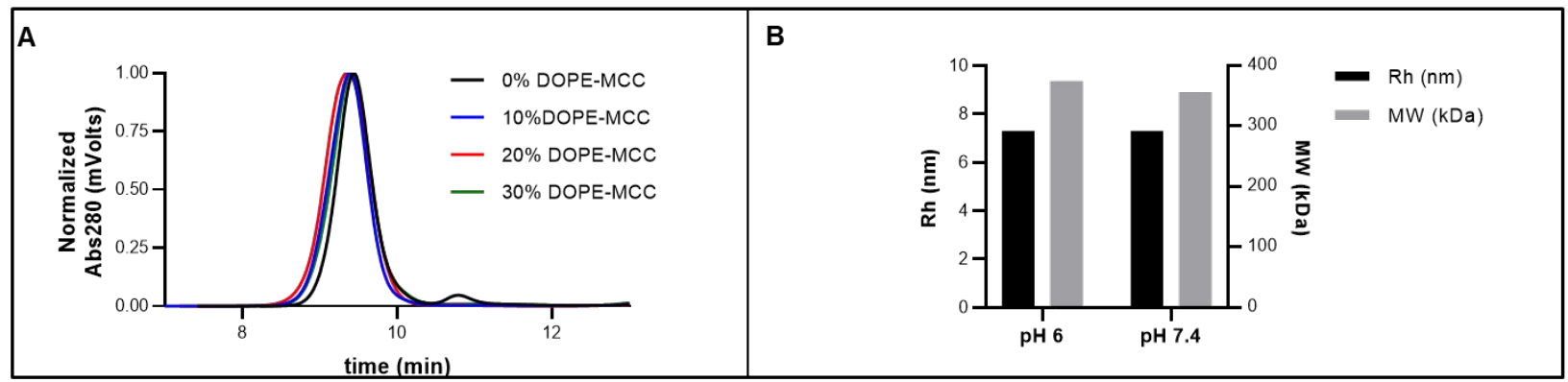

Figure S2. Effect of DOPE-MCC on NLP assembly. A. SEC analysis of NLPs assembled with increasing molar concentrations of DOPE-MCC. B. $\mathrm{MW}$ and Rh analysis of NLP assembled under crosslinking conditions ( $\mathrm{pH} \mathrm{7.4)} \mathrm{and} \mathrm{non-crosslinking} \mathrm{conditions} \mathrm{(pH} \mathrm{6).}$ 